\title{
Absolute Stability of Nonlinear Systems with Piecewise Linear Sector Condition
}

\author{
Amin Rezaei \\ Electrical Engineering Department, Faculty of \\ Engineering, University of Bonab, Bonab, Iran
}

\author{
Farzad Hashemzade \\ Control Engineering Department, Faculty of \\ Electrical and Computer Engineering, University of \\ Tabriz, Tabriz, Iran
}

\begin{abstract}
The circle theorem is one of the most important methods for study of absolute stability of nonlinear systems. In circle theorem, it is assumed that the nonlinearity term is located between linear bounds of a typical sector. In this paper the absolute stability of nonlinear systems with generalized sector condition is studied in which the bounds of the sector are piecewise linear in general. Therefore this method could be applied to the sector with nonlinear bounds. In this paper using modified Nyquist criterion, it is proved that the study of absolute stability of a nonlinear system using circle theorem could be reduced to the study of stability of equalized linear system. The aim of this paper is to define a pseudo circle region that the Nyquist plot of the linear system with nonlinearity in sector condition with piecewise bounds doesn't have any intersection with that region and encircle it $m$ times in the counterclockwise direction where $m$ is the number of poles of linear part of the system with positive real parts.
\end{abstract}

\section{Keywords}

Circle theorem, Lemma, Non linear systems

\section{INTRODUCTION}

Many nonlinear physical systems can be represented as a feedback connection of a linear dynamical system and a nonlinear element, as shown in Fig1. The absolute stability of the feedback system in Fig1 is a classical problem in control theory. It has been addressed extensively in the nonlinear systems and control literature ([1], [2], [3]). The usual tools for absolute stability analysis include Popov criterion and circle criterion. With the advancement of robust control theory in the 1980s and 1990s, some close relationship between robustness and absolute stability was established in [4] and robustness analysis tools were applied to deal with absolute stability problems ([3], [4], [5], [6]). Among these tools, circle criterion appears to be the most popular.

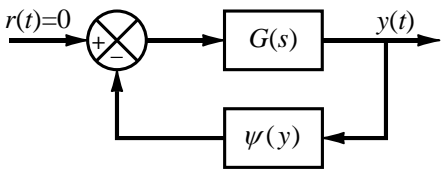

Fig 1: Representation of a nonlinear physical system.

It is assumed the transfer function matrix of the linear system is given by $G(s)=C(s I-A)^{-1} B$ which is a square strictly proper transfer function and $u=\psi(y)$ in which $\psi$ is a memory less, possibly time varying nonlinearity which is piecewise continuous in $\mathrm{t}$ and locally Lipschitz in $\mathrm{y}$ and In this case, $\psi:[0, \infty) \times R \rightarrow R$ satisfies a sector condition (or is a sector nonlinearity) if there are constants $\alpha, \beta, a$ and $b$ (with $\alpha<\beta$ and $a<0<b$ ) such that $\forall y \in[a, b]: \alpha y^{2} \leq y \psi(y) \leq \beta y^{2}$

If (1) holds for all $y \in(-\infty, \infty)$ is said that the sector condition holds globally. This sector condition is shown in Fig2.

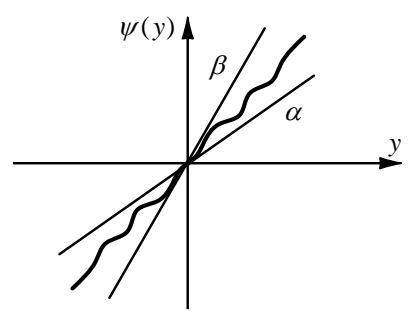

Fig 2: Sector condition $(\alpha, \beta)$

The stability of the above system is studied using circle theorem is as following [3]:

Circle Theorem: Consider a scalar system of the form $G(s)=C(s I-A)^{-1} B \quad$ where $\quad\{A, B, C\} \quad$ is a minimal realization of $G(s)$ and $\psi$ satisfies the sector condition (1) globally. Then the system is absolutely stable if one of the following conditions is satisfied, as appropriate:

1. If $0<\alpha<\beta$, the Nyquist plot of $G(j \omega)$ does not enter the disk $D(\alpha, \beta)$ in Fig3 and encircles it $m$ times in the counterclockwise direction, where $m$ is the number of poles of $G(s)$ with positive real parts.

2. If $0=\alpha<\beta, G(s)$ is Hurwitz and the Nyquist plot of $G(j \omega)$ lies to the right of the vertical line defined by $\operatorname{Re}(s)=-1 / \beta$.

3. If $\alpha<0<\beta, G(s)$ is Hurwitz and the Nyquist plot of $G(j \omega)$ lies in the interior of the disk $D(\alpha, \beta)$ in Fig3.

If the sector condition (1) is satisfied only on an interval $[a, b]$ then the foregoing conditions ensure that the system is absolutely stable with a finite domain.

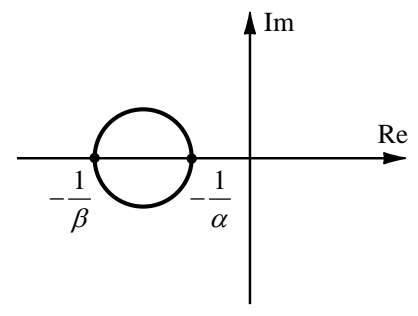

Fig 3: disk $D(\alpha, \beta)$.

In this paper the absolute stability of nonlinear systems with generalized sector condition is studied in which the bounds of the sector are piecewise linear in general. Therefore this 
method could be applied to the sector with nonlinear bounds. In this paper using modified Nyquist criterion, it is proved that the study of absolute stability of a nonlinear system using circle theorem could be reduced to the study of stability of linear system. The aim of this paper is to define a pseudo circle region that the Nyquist diagram of the linear system with nonlinearity in sector condition with piecewise bounds doesn't have any intersection with that region and turn it equal to the number of its unstable poles.

In the remind of the paper, analysis of absolute stability using modified Nyquist criteria studied in section 2 and Circle criteria for non-typical sector condition is described in section 3. In section 4, Circle criteria for piecewise linear sector condition is studied and the simulation results in section 5, shown the beneficiary of this new method.

\section{ANALYSIS OF ABSOLUTE STABILI- TY USING MODIFIED NYQUIST CRIT-ERIA}

In this paper, it is resulted that the absolute stability of a nonlinear system using circle theorem could be reduced to the study of stability of linear system. In theorem I, will be proved that there is a linear system $G_{t}(s)$ which is equal to the system $G(s)$ with nonlinearity in sector condition $(\alpha, \beta)$, in the sense of stability.

In other words, the stability of $G(s)$ with nonlinearity in sector condition $(\alpha, \beta)$ could be studied using the stability of a linear system $G_{t}(s)$ and vice versa. To prove theorem I, two lemmas are studied in following.

Lemma I: If the Nyquist plot of $G(s)$ encircles the Nyquist plot of $-1 / k(s) m$ times ( $m$ is the number of poles of $G(s)$ with positive real parts) then the feedback system Fig4 is asymptotically stable.

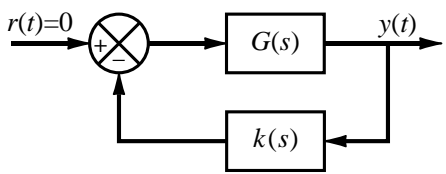

Fig 4:the feedback interconnection of the system $G(s)$ and $k(s)$

Proof of the Lemma I: According to the modified Nyquist criteria, for the stability of $1+k_{i} G(s)=0$, the Nyquist plot of $G(s)$ should encircle the point $-1 / k_{i}, m$ times in counterclockwise in which $k_{i}$ could be a complex number [3]. Therefore it is possible to say that, the system Fig4 is stable if the characteristic equation $1+k_{i} G(s)=0$ be stable for all $k_{i}$, where $k_{i}=k\left(j \omega_{i}\right)$ for all $\omega_{i} \in(-\infty, \infty)$. It is important to say that, the Nyquist plot of $-1 / k(s)$ is the combination of the points $-1 / k_{i}$ in s-plane.

Lemma II: if the system of Fig4 be asymptotically stable then the Nyquist plot of $G(s)$ encircle the Nyquist plot of $-1 / k(\gamma s), \mathrm{m}$ times and there isn't any intersection between these Nyquist plots where $\mathrm{m}$ is the number of poles of $G(s)$ with positive real parts and $\gamma>0$.

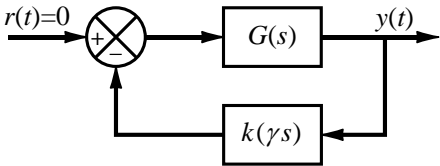

Fig 5: the feedback interconnection of the system $G(s)$ and $\boldsymbol{k}(\gamma s)$
Proof of the Lemma II: According to the fact that, the Nyquist plot of $-1 / k(\gamma s)$ is equal to the Nyquist plot $-1 / k(s)$ so using lemma I, the Nyquist plot of $G(s)$ encircle the Nyquist plot of $-1 / k(\gamma s), m$ times. To prove the lack of intersection, using reductio ad adsurdum method, is assumed that the Nyquist plots of $G(s)$ and $-1 / k(\gamma s)$ have at least one intersection at point $\mathrm{P}$ where the point $\mathrm{P}$ is the map of $G\left(j \omega_{1}\right)$ and $-1 / k\left(\gamma j \omega_{2}\right)$. If $\omega_{1}=\omega_{2}$ then $1+k\left(j \omega_{1}\right) G\left(j \omega_{2}\right)=0$ which means that the system is not asymptotically stable which is in contradiction with assumption of lemma II. So the assumed intersection should be happen in $\omega_{1} \neq \omega_{2}$.

At point $\mathrm{P}$, we have

$G\left(j \omega_{1}\right)=-\frac{1}{k\left(j \omega_{2}\right)} \stackrel{\gamma_{0}=\omega_{2} / \omega_{1}}{=} G\left(j \omega_{1}\right)=-\frac{1}{=}=$

Therefore there are $\gamma_{0}$ and $\omega_{1}$ in such a way that $1+$ $k\left(\gamma_{0} s\right) G(s)=0$ is not asymptotically stable which contradicts with the asymptotically stability assumption of lemma II.

Theorem I: the system $G(s)$ with nonlinearity in sector $(\alpha, \beta)$ is stable iff the linear feedback system $\mathrm{G}(\mathrm{s})$ and $k(\gamma s)$ be stable where

$-\frac{1}{k(s)}=-\frac{\alpha s+\beta}{\alpha \beta(s+1)}$

Proof of the Theorem I: Assume that the system Fig6.a is asymptotically stable. According to the circle criteria, the Nyquist diagram of $G(s)$ encircle the disk $D(\alpha, \beta)$ in Fig3, $m$ times in which $m$ is the number of poles of $G(s)$ with positive real parts. According to this fact that the Nyquist plot of $1 / k(\gamma s)$ is equal to the circle in Fig3, so to have asymptotically stability of system in Fig6.a, the Nyquist plot of $G(s)$ should encircle the Nyquist plot of $1 / k(\gamma s)$ for all $\gamma>0, \mathrm{~m}$ times. Because of the equality between the Nyquist plot of $-1 / k(\gamma s)$ and $-1 / k(s)$, using the lemma I the system in Fig6.b is asymptotically stable.

Assume that the system in Fig6.b is asymptotically stable, using lemma II the Nyquist plot of $G(s)$ doesn't have any intersection with the Nyquist plot of $-1 / k(\gamma s)$ and encircle it $\mathrm{m}$ times. Because of the similarity between the Nyquist plot of $-1 / k(\gamma s)$ and the circle in Fig3, the Nyquist plot of $G(s)$ doesn't have any intersection with the circle in Fig3 and encircle it $m$ times. Therefore the system in Fig6.a is asymptotically stable.

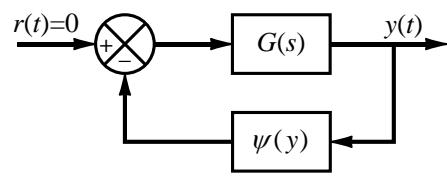

Fig 6.a: the feedback interconnection of the system $\boldsymbol{G}(\boldsymbol{s})$ and nonlinearity $\psi(y)$

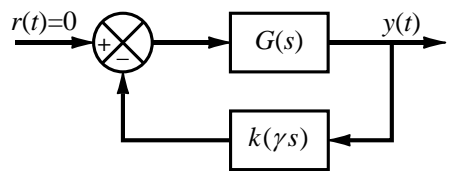

Fig 6.b: the feedback interconnection of the system $G(s)$ and $k(\gamma s)$ 


\section{CIRCLE CRITERIA FOR NONTYPIC-AL SECTOR CONDITION}

In this part we want to study the circle theorem for the system with non-typical sector shown in Fig7.a. To do this we should convert the system in Fig7.a to typical sector problem shown in Fig7.b in which the definition of d0 is as follows:

$d_{o}(t)= \begin{cases}-b & ; y_{1}(t) \geq b \\ -y_{1}(t) & ;-b \leq y_{1}(t)<b \\ b & ; y_{1}(t)<-b\end{cases}$

After some simplifications, the system shown Fig7.b is converted to the system in Fig8.

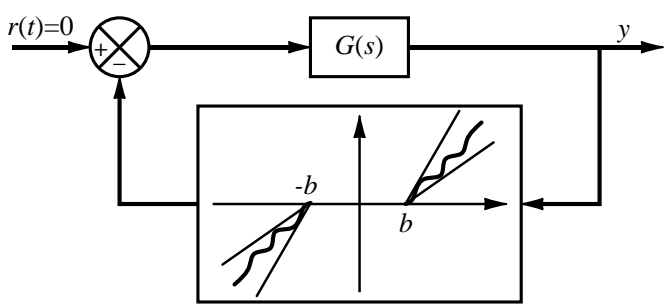

Fig 7.a: the linear system $G(s)$ and nonlinearity in nontypical sector condition.

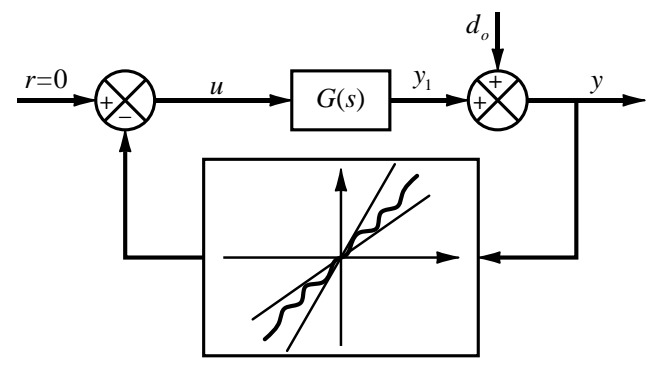

Fig 7.b: the linear system G(s) and nonlinearity in typical sector condition with input $\mathrm{do}$

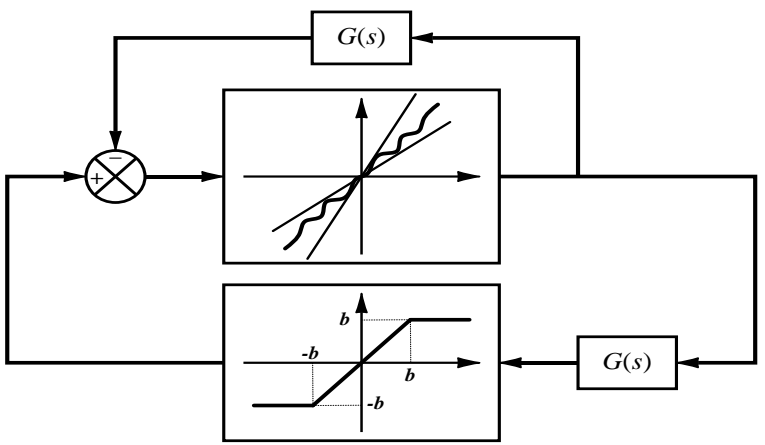

Fig 8: the simplified system of Fig 7.b

According to the theorem I, the feedback connection between $G(s)$ and typical sector $(\alpha, \beta)$, could be modeled as $G_{t}(s)$ (the transfer function of the system in Fig6.b) in the sense of stability. Therefore the system in Fig8 could be simplified to the system in Fig9, where $G_{t}(s)$ is as follows:

$G_{t}(s)=\frac{G(s) k(\gamma s)}{1+G(s) k(\gamma s)}$

$G(s)=\frac{G_{t}(s)}{k(\gamma s)\left[\left(1-G_{t}(s)\right)\right]}$

The result of above simplifications is reduced to the stability study of the system in Fig9. The nonlinearity term in Fig9 could be modeled as a typical sector $(0,1)$. Therefore the stability of the system in Fig9 is equal to the stability of the system $G_{t}(s)$ with sector $(0,1)$.

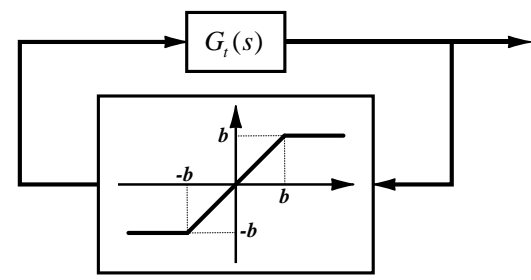

Fig 9: the simplified system of Fig8

Using the circle criteria, the system in Fig9 is stable iff the Nyquiast plot of $G_{t}(s)$ does not enter the region $\operatorname{Re}(s)<-1$. The region $\operatorname{Re}(s)<-1$, is equal to the Nyquiast plot of $L(s)=-1-1 / s$

According to (5), it is possible to define a bilinear mapping that maps the Nyquist plot of $G_{t}(s)$ to the Nyquist plot of $G(s)$. The applying result of this mapping to the region $\operatorname{Re}(s)<-1$ generates a region $\mathrm{M}(\mathrm{s})$ that the Nyquist plot of $G(s)$ encircle it $\mathrm{m}$ times and doesn't have any intersection with it. The above described mapping is as follows:

$W=\frac{Z}{k(\gamma s)(1-Z)}$

Also the mapping result of region $\operatorname{Re}(s)<-1$, is in following form.

$M(s)=\frac{L(s)}{k(\gamma s)(1-L(s))}=-\frac{(\alpha \gamma s+\beta)(s+1)}{\alpha \beta(\gamma s+1)(2 s+1)}$

\section{CIRCLE CRITERIA FOR PIECEWISE LINEAR SECTOR CONDITION}

In Fig10, a sector with piecewise linear bounds is shown. This sector is the combination of two typical and non-typical sectors Fig 11 . To stability analysis of system shown in Fig 10 with piecewise sector bounds, it is possible to convert it to the connection shown in Fig12.a in which a general sector could be replaced as a summation of two typical and non-typical sectors.

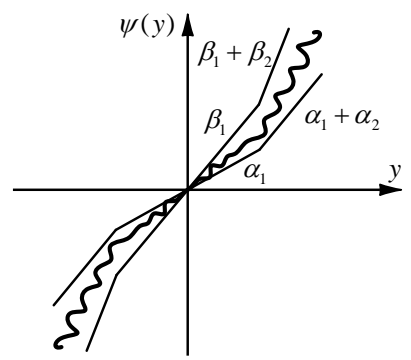

Fig 10: a sector with piecewise linear bounds
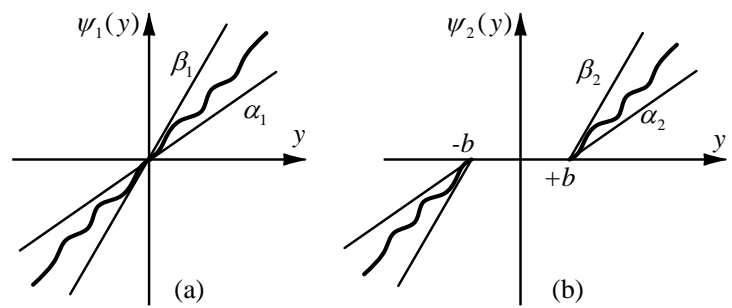

(b)

Fig 11: typical and non-typical sectors combined to achieve the sector shown in Fig10 
It is easy to see from Fig12.a that, according to the theorem I, the internal nonlinear loop could be replaced with $G_{t}(s)$ in the sense of stability which is shown in Fig12.b. Therefore the stability analysis of the system Fig12.a could be studied using the stability analysis of the system Fig12.b.

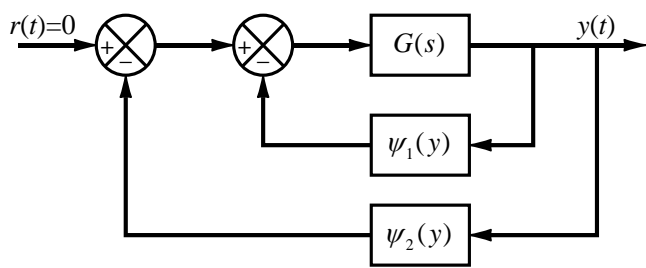

Fig12.a: $G(s)$ with typical sector $\psi_{1}(s)$ and non-typical sector $\psi_{2}(s)$

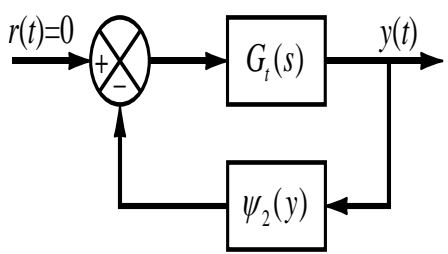

Fig 12.b: $G_{t}(s)$ with non-typical sector $\psi_{2}(s)$

\section{SIMULATION RESULTS}

In this section, the result of the new method in absolute stability analysis is shown and advantages of its compared with circle theorem. As shown in Fig13, it is assumed that, there exist a feedback connection between a linear system $G(s)$ and a deadzone, where $G(s)$ is as following:

$G(s)=\frac{(s+1)^{2}}{s(s+3)(s+.0001)}$

To study absolute stability of this system using circle theorem, the nonlinearity deadzone should be modeled as a sector with $\alpha=0$ and $\beta=1$. Using circle theorem, the system is absolutely stable if the Nyquist plot of $G(s)$ be in the right side of the line $\operatorname{Re}(s)=-1$. As shown in the Fig14, the Nyquist plot of G(s) is not on the right side of the $\operatorname{Re}(s)=$ -1 . Therefore it is not possible to analysis the absolute stability of the system using circle theorem.

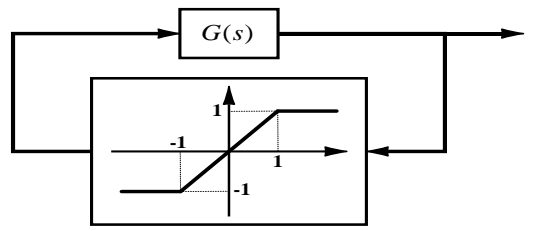

Fig 13: feedback connection between a linear system $G(s)$ and a deadzone.

Using the generalized circle theorem introduced in this paper, it is possible to analysis the absolute stability of the system. Deadzone could be modeled as a non-typical sector with $\alpha=\beta=1$ which is introduced in the system in Fig7.a. In section 3 , it is proved that the system is stable if the Nyquist plot of $G(s)$ doesn't enter to the Nyquist plot of $M(s)$ in (7) and doesn't encircle it.

In Fig14, the Nyquist plot of $M(s)$ is shown which is similar to a circle. It is obvious to see that, the Nyquist plot of $G(s)$ doesn't encircle the Nyquist plot of $M(s)$ and doesn't enter to it. So using the result of this paper, we could say that the system $G(s)$ with a deadzone in feedback in Fig13 is absolutely stable.

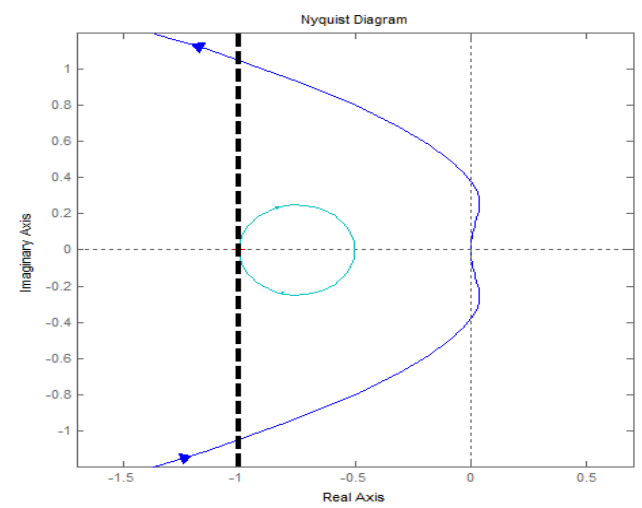

Fig 14: the Nyquist plot of $G(s)$, the Nyquist plot of $M(s)$ and the line $\operatorname{Re}(s)=-1$

\section{CONCLUSION}

This paper presents a new method for study of stability of a nonlinear system using generalized circle criterion. The circle criterion is generalized and using this generalization, modeled a linear system with nonlinearity in sector $(\alpha, \beta)$ by a equalized linear feedback system, and studied the stability of this linear feedback system instead of stability of the nonlinear system. The brilliant result of this paper is to generate a pseudo circle region that the stability of a system with piecewise linear bounds on its sector could be achieved using the analysis of this pseudo circle region and the Nyquist plot of linear part of the system.

Using the method introduced in this paper, can be studied the absolute stability of the systems with nonlinearity on their sector bounds which could be achieved by the approximation of nonlinear bounds of the sector by piecewise linear bounds.

\section{REFERENCES}

[1] M. A. Aizerman and F. R. Gantmacher, Absolute Stability of Regulator Systems. San Francisco, CA: Holden-Day, 1964.

[2] J. C. Hsu and A. U. Meyer, Modern Control Principles and Applications. New York: McGraw-Hill, 1968.

[3] H. Khalil, Nonlinear Systems. Upper Saddle River, NJ: Prentice-Hall, 1996.

[4] Y. S. Chou, A. L. Tits, and V. Balakrishnan, "Stability multipliers and upper bounds: Connections and implications for numerical verification of frequency domain conditions," IEEE Trans. Automat. Contr., vol. 44, pp. 906-913, June 1999.

[5] K. Gu, "absolute stability of systems under block diagonal memoryless uncertainties," Automatica, vol. 31, no. 4, pp. 581-584, 1995.

[6] A. Megretski and A. Rantzer, "system analysis via integral quadratic constraints," IEEE Trans. Automat. Contr., vol. 42, pp. 819-830, May 1997.

[7] Amin Rezaei, Linear Control Systems, 6 ${ }^{\text {th }}$, Pouran Pazhuhesh Pub., Tehran, Iran, 2015. 